\title{
Variability of laying hen behaviour depending on the breed
}

\author{
Agnieszka Kozak ${ }^{1}{ }^{1 *}$, Kornel Kasperek ${ }^{1}$, Grzegorz Zięba ${ }^{1}$, and Iwona Rozempolska-Rucińska ${ }^{1}$
}

\section{* Corresponding Author: Agnieszka Kozak Tel: +48-504-505-885, Fax: +48-81-533-35-49, E-mail: agnieszka.kozak@up.lublin.pl}

'Institute of Biological Basis of Animal Production, University of Life Sciences in Lublin, Lublin 20-950, Poland

ORCID

Agnieszka Kozak

https://orcid.org/0000-0002-3272-9862

Kornel Kasperek

https://orcid.org/0000-0003-4305-8168

Grzegorz Zięba

https://orcid.org/0000-0003-0358-5692

wona Rozempolska-Rucińska

https://orcid.org/0000-0001-5025-0225

Submitted Aug 31, 2018; Revised Oct 1, 2018; Accepted Nov 29, 2018
Objective: For many generations, most species of farm animals have been subjected to intense and strictly targeted selection for improvement of their performance traits. This has led to substantial changes in animal anatomy and physiology, which resulted in considerable differences between the current animal breeds and their wild ancestors. The aim of the study was to determine whether there is breed-specific variability in behaviour as well as differences in emotional reactivity and preferences of laying hens.

Methods: The investigations involved 50 Green-legged Partridge, 50 Polbar, and 50 Leghorn hens. All birds were kept in the same conditions, and the behavioural tests were carried out at 30 weeks of age. We used the tonic immobility test and a modified open-field test including such objects as water, commercial feed, feed enriched with cereal grains, finely cut straw, and insect larvae, a sandpit, a mirror, and a shelter imitating a hen nest.

Results: The research results demonstrate that the birds of the analysed breeds differ not only in the excitability and emotional reactivity but, importantly, also in the preferences for environment-enriching elements. Ensuring hen' well-being should therefore be based on environmental modifications that will facilitate acquisition of essential elements of chickens' behaviour. The greatest emotional reactivity was found in the Leghorn breed, which may be a result of correlated selection aimed at an increase in chicken productivity.

Conclusion: The differences in the behaviour of the birds from the analysed breeds indicate that laying hens cannot be regarded as one group of animals with the same environmental requirements.

Keywords: Laying Hen; Behavioural Test; Breed

\section{INTRODUCTION}

Most farm animal species have long been subjected to intense and strictly targeted selection for improvement of their performance traits. Programs implemented in chicken breeding are mainly focused on increasing weight gain, laying performance, or internal and external egg quality traits. This has led to substantial changes in the anatomy and physiology of this species, which resulted in considerable differences between birds reared currently and their wild ancestors, also in terms of their behaviour. Natural hens' behaviour represents a repertoire of their ancestors' behaviours, provided that their rearing conditions allow demonstration of such behaviours [1]. Behavioural problems arise when chickens are motivated to show certain behaviours but they are unable to express them due to limitations such as the size of the cage or absence of enrichment elements. Consequently, other variants of behaviour arise and can often lead to behavioural disorders, e.g. feather plucking. Behavioural patterns depend not only on birds' habitat and experiences but also on the genetic background, environmental conditions prevailing during embryonic development, and epigenetic effects $[2,3]$. Therefore, it seems that each breed of laying hens reared on farms can exhibit diverse behavioural needs for maintenance of homeostasis of the organism. However, the differences in behavioural needs between chicken genotypes are not considered during adjustment of 
rearing standards to ensure bird welfare. Therefore, standardisation of the conditions for rearing laying hens on farms does not necessarily improve the welfare of all breeds.

The aim of the study was to determine whether there is variability in behaviour as well as differences in emotional reactivity and preferences of laying hens depending on the breed.

\section{MATERIALS AND METHODS}

\section{Birds and husbandry}

All procedures employed during the research were approved by the II Local Ethics Committee for Animal Testing at the University of Life Sciences in Lublin, Poland (Approval No. 69/2017 of 28 September 2017). The investigations involved 150 birds, including 50 Green-legged Partridge (Zk), 50 Polbar $(\mathrm{Pb})$, and 50 Leghorn $(\mathrm{LG})$ hens. The Green-legged Partridge is a native chicken breed described at the end of the 19th century. It is often reared on organic farms [4]. The hens are perfectly adapted to the conditions of extensive rearing in free-range systems. Polbar is a Polish autosexing synthetic breed produced by mating Green-legged Partridge hens with Plymouth Rock cocks. The Polbar breed traits were conserved in the 1950s. It should be noted that both breeds ( $\mathrm{Zk}$ and $\mathrm{Pb}$ ) are reared in closed, unselected populations in accordance with the phenotypic and functional pattern developed for protection of animal genetic resources [5]. The Leghorn is one of the most popular laying hen breeds in Europe, which is especially adapted to intensive breeding and subjected to intensive selection towards performance traits [6].

All birds were kept in the same farm building on straw bedding, which guaranteed identical rearing conditions. The hens were 30 weeks old at the time of the experiment. The birds were kept in 6 group boxes, with 25 females of one line in each at a density of $0.3 \mathrm{~m}^{2} /$ bird. All boxes were equipped with nipple drinkers, feeders, and nests, and with 16 hours of light each day. The birds were fed and maintained according to standard breeding requirements for poultry. The tests were carried out from 8.00 to $15.00 \mathrm{~h}$ for 6 days ( 1 day $=1$ box).

\section{Open-field test}

All birds were subjected to the open-field test individually [7] for $10 \mathrm{~min}$. The test was carried out in an observation box with a $1.25 \times 1.25 \mathrm{~m}$ floor divided into 25 squares with an area of $25 \times 25 \mathrm{~cm}$ each. A camera viewing the entire area available for the birds was mounted above the box and each test was recorded. The open-field test was modified, i.e. additional elements enriching the environment were placed in the box. These elements included a container with water, a container with commercial feed, a container with feed supplemented with cereal grains, finely cut straw, and insect larvae, a sandpit, a mirror, and a shelter imitating hen nests. The birds were placed in the central point of the box to keep the same distance from the enriching objects. The analysis of film recordings was carried out to determine the duration of animal's exploration of the objects and locomotion throughout the test measured by means of a hand-held stopwatch. Such behaviours as vocalisation, defecation, comfort behaviour, interest in the floor, and shake-off were noted. The analysed elements and the measurement procedure are presented in Table 1.

\section{Tonic immobility}

Immediately after the open-field test, the tonic immobility test described by Jones [8] was applied. The birds were immobilised by being placed in a special cradle [6] on the back. Their sternum was pressed gently in such a position that the head could hang backwards freely. The latency of tonic immobility

Table 1. Assessment of hen behaviour during the enriched open-field test

\begin{tabular}{ll}
\hline Object/Behaviour & Measurement procedure \\
\hline Latency of undertaking activity & $\begin{array}{l}\text { Time between placing the bird on the floor of the experimental box and the first movement, e.g. Head movements, looking } \\
\text { around, but no locomotor activity (s) }\end{array}$ \\
$\begin{array}{l}\text { Latency of undertaking exploration } \\
\text { Time of locomotion }\end{array}$ & $\begin{array}{l}\text { Time between placing the bird on the floor in the experimental box and the beginning of locomotor activity (s) } \\
\text { Time spent by the hen on locomotion throughout the test, excluding the activity time;measured in seconds (s) }\end{array}$ \\
Exploration area & Time spent by the hen observing and showing interest in the objects; measured in seconds (s) \\
Water (W) & Number of squares covered by the tested hen \\
Commercial feed (CF) & Time spent on exploration of the object or using its resources; measured in seconds (s) \\
Enriched feed (EF) & Time spent on exploration of the object or using its resources; measured in seconds (s) \\
Sandpit (SA) & Time spent on exploration of the object or using its resources; measured in seconds (s) \\
Mirror (M) & Time spent on exploration of the object or using its resources; measured in seconds (s) \\
Shelter (S) & Time spent on exploration of the object or pecking at own reflectionin the mirror; measured in seconds (s) \\
Floor (F) & Time spent on exploration of the object or staying inside; measured in seconds (s) \\
Vocalisation & Time spent on exploration of the floor; measured in seconds (s) \\
Defecation & Assessed in a 0-1 point scale, where 0 denoted no vocalisation, 1-presence of vocalisation \\
Comfort behaviour & Assessed in a 0-1 point scale, where 0 denoted no defecation, 1 - presence of defecation \\
Shake-off & Assessed in a 0-1 point scale, where 0 denoted no comfort behaviour, 1 - presence of comfort behaviour \\
\hline
\end{tabular}


was measured. Upon the tonic immobility, the experimenter released the pressure and latency to the first head movement and straightening (commonly referred to as the duration of tonic immobility test [TI1]) was measured (tonic immobility 2 [TI2]) [8].

\section{Statistical analysis}

Since the recorded traits do not have a normal distribution, the data were rank-transformed [9] Bonferroni-corrected multiple comparisons of the estimations of differences in the analysed traits between the breeds were analysed with 2-factor models considering the effect of the genetic group and the object of interest. The analyses were performed with the use of the GLIMMIX procedure in the SAS version 9.4 program (SAS Institute, Cary, NC, USA). The probability of the occurrence of a specific reaction depending on the bird breed was determined as well. The significance of the differences was verified by the analysis of variance and the least square method considering the fixed effect of the genetic group in the model (SAS Institute, USA).

The results are presented as arithmetic means (Tables 2, 3) to facilitate biological interpretation.

\section{RESULTS}

\section{Open field test and tonic immobility}

An almost two-fold longer TI1 was noted in the LG hens than in the $\mathrm{Zk}$ and $\mathrm{Pb}$ breeds (Table 2) and the differences were statistically significant (Table 4). The TI2 was almost three-fold shorter in $\mathrm{Pb}$ than in $\mathrm{Zk}$, with statistical significance of the differences (Table 4). There were no statistically significant differences in the latency of undertaking activity and exploration and in the total duration of locomotion and examination of the objects between the tested chicken breeds (Table 4). However, the number of squares covered by the LG hens was several-fold greater than that in the $\mathrm{Pb}$ and $\mathrm{Zk}$ breeds.

\section{Defecation and vocalisation}

A highly significant difference was observed in the probability of occurrence of defecation, self-grooming behaviour, and shake-off behaviour between the LG hens and the $\mathrm{Zk}$ and $\mathrm{Pb}$ hens. The probability of vocalisation was several-fold higher in $\mathrm{Pb}$ and $\mathrm{LG}$ than in $\mathrm{Zk}$ (Table 5).

\section{Elements enriching the environment}

The $\mathrm{Pb}$ birds showed the greatest interest in the water, as they spent nearly twice as much time at the drinker than the $\mathrm{Zk}$ and LG hens; however, these differences were not statistically significant (Tables 3,6 ). The longest time spent on eating the commercial feed was exhibited by the LG chickens. In turn, the greatest interest in the enriched feed was noted in the case of the $\mathrm{Zk}$, which also exhibited the greatest interest in this object (Table 3). The shelter was an enrichment element that received the lowest interest from the $\mathrm{Pb}$ hens, and the differences were statistically significant in comparison with the other breeds (Tables 3,6). The mirror aroused the greatest interest among the LG birds, which devoted over a 5-fold and 3-fold longer time than the $\mathrm{Zk}$ and $\mathrm{Pb}$ breeds, respectively (Table 3 ). There

Table 2. Mean level of traits defining the emotional reactivity of birds depending on the breed

\begin{tabular}{|c|c|c|c|c|c|}
\hline Breed & Variable & Mean & Standard error & Min & Max \\
\hline \multirow[t]{7}{*}{ Leghorn } & Latency of tonic immobility & 60.4 & 59.8 & 0 & 322 \\
\hline & Duration of tonic immobility & 28.5 & 52.4 & 0 & 278 \\
\hline & Latency of activity & 81.6 & 137.9 & 1 & 600 \\
\hline & Latency of exploration & 284.5 & 230.3 & 2 & 600 \\
\hline & Time of examination of objects & 518.4 & 137.9 & 0 & 599 \\
\hline & Duration of locomotion & 316.4 & 229.7 & 0 & 598 \\
\hline & Explored area & 25.2 & 33.9 & 0 & 110 \\
\hline \multirow[t]{7}{*}{ Polbar } & Latency of tonic immobility & 39.3 & 52.2 & 0 & 247 \\
\hline & Duration of tonic immobility & 11.7 & 24.9 & 0 & 111 \\
\hline & Latency of activity & 92.0 & 197.9 & 1 & 600 \\
\hline & Latency of exploration & 257.0 & 237.8 & 1 & 600 \\
\hline & Time of examination of objects & 508.0 & 197.9 & 0 & 599 \\
\hline & Duration of locomotion & 343.0 & 237.8 & 0 & 599 \\
\hline & Explored area & 6.0 & 8.7 & 0 & 44 \\
\hline \multirow[t]{7}{*}{ Green-legged Partridge } & Latency of tonic immobility & 35.0 & 27.7 & 0 & 111 \\
\hline & Duration of tonic immobility & 36.2 & 53.0 & 0 & 217 \\
\hline & Latency of activity & 47.5 & 141.8 & 1 & 600 \\
\hline & Latency of exploration & 303.3 & 244.4 & 1 & 600 \\
\hline & Time of examination of objects & 552.5 & 141.8 & 0 & 599 \\
\hline & Duration of locomotion & 297.5 & 244.3 & 0 & 599 \\
\hline & Explored area & 5.2 & 8.1 & 0 & 44 \\
\hline
\end{tabular}


Table 3. Mean duration of interest in the objects depending on the breed

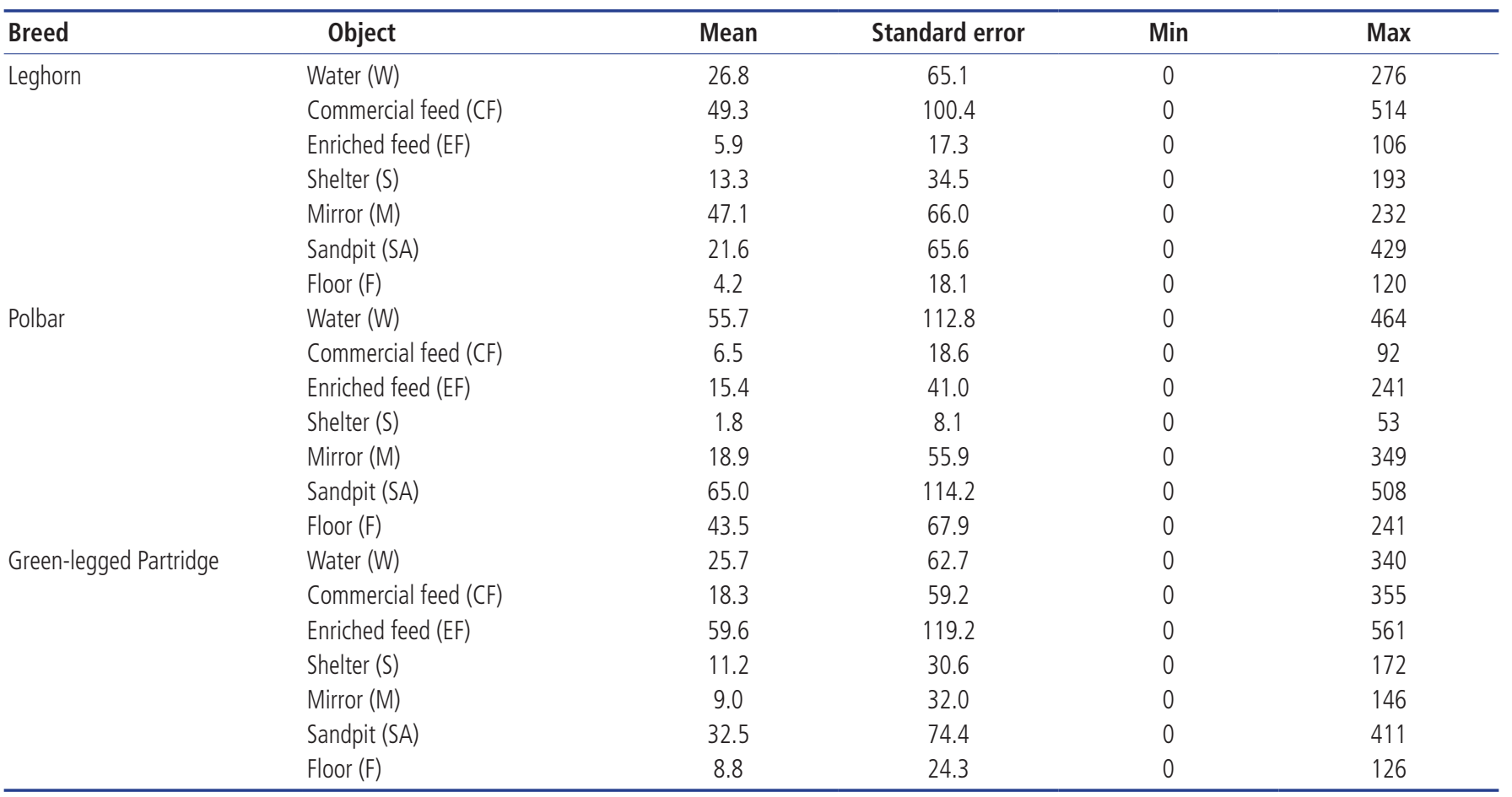

were no differences between the genetic groups in the time devoted to examination of the floor and in the interest in the sandpit (Table 6).

\section{DISCUSSION}

A major hen welfare-related problem in modern industrial poultry farming is the lack of adjustment of rearing conditions

Table 4. Estimators of differences in traits defining the emotional reactivity of birds depending on the breed, their significance and confidence interval

\begin{tabular}{|c|c|c|c|c|c|c|c|}
\hline \multirow{2}{*}{$\frac{\text { Type of test }}{\text { Tonic immobility }}$} & \multirow{2}{*}{$\begin{array}{l}\text { Trait (unit of measurement) } \\
\text { Latency of tonic immobility (s) }\end{array}$} & \multicolumn{2}{|c|}{ Breed } & \multirow{2}{*}{$\begin{array}{c}\text { Estimate } \\
0.42\end{array}$} & \multirow{2}{*}{$\begin{array}{r}\operatorname{Pr}>|t| \\
0.031\end{array}$} & \multirow{2}{*}{$\begin{array}{c}\text { Adjust lower } \\
-0.046\end{array}$} & \multirow{2}{*}{$\begin{array}{c}\text { Adjust uppe } \\
0.887\end{array}$} \\
\hline & & $L G$ & $\mathrm{~Pb}$ & & & & \\
\hline & & $L G$ & Zk & 0.52 & 0.007 & 0.057 & 0.991 \\
\hline & & $\mathrm{Pb}$ & Zk & 0.10 & 0.592 & -0.363 & 0.570 \\
\hline & Duration of tonic immobility (s) & LG & $\mathrm{Pb}$ & 0.35 & 0.067 & -0.110 & 0.818 \\
\hline & & $L G$ & Zk & -0.17 & 0.381 & -0.632 & 0.296 \\
\hline & & $\mathrm{Pb}$ & Zk & -0.52 & 0.007 & -0.986 & -0.058 \\
\hline \multirow[t]{15}{*}{ Open field test } & Latency of activity (s) & LG & $\mathrm{Pb}$ & 0.13 & 0.489 & -0.332 & 0.598 \\
\hline & & LG & Zk & 0.28 & 0.154 & -0.190 & 0.740 \\
\hline & & $\mathrm{Pb}$ & Zk & 0.14 & 0.461 & -0.323 & 0.607 \\
\hline & Latency of exploration (s) & LG & $\mathrm{Pb}$ & 0.07 & 0.703 & -0.348 & 0.478 \\
\hline & & $L G$ & Zk & -0.12 & 0.468 & -0.537 & 0.289 \\
\hline & & $\mathrm{Pb}$ & Zk & -0.19 & 0.269 & -0.602 & 0.224 \\
\hline & Explored area & $L G$ & $\mathrm{~Pb}$ & 0.68 & 0.000 & 0.231 & 1.130 \\
\hline & & LG & Zk & 0.76 & 0.000 & 0.312 & 1.210 \\
\hline & & $\mathrm{Pb}$ & Zk & 0.08 & 0.666 & -0.369 & 0.530 \\
\hline & Duration of examination of objects (s) & $L G$ & $\mathrm{~Pb}$ & -0.32 & 0.091 & -0.774 & 0.136 \\
\hline & & LG & Zk & -0.07 & 0.698 & -0.528 & 0.382 \\
\hline & & $\mathrm{Pb}$ & Zk & 0.25 & 0.192 & -0.209 & 0.701 \\
\hline & Duration of locomotion (s) & LG & $\mathrm{Pb}$ & -0.27 & 0.165 & -0.748 & 0.201 \\
\hline & & LG & Zk & -0.06 & 0.743 & -0.539 & 0.410 \\
\hline & & $\mathrm{Pb}$ & Zk & 0.21 & 0.288 & -0.266 & 0.684 \\
\hline
\end{tabular}

LG, Leghorn; Pb, Polbar; Zk, Green-legged Partridge. 
Table 5. Probability of occurrence of reactions depending on the breed

\begin{tabular}{|c|c|c|c|c|c|c|c|}
\hline \multirow{2}{*}{ Type of test } & \multirow{2}{*}{ Trait (unit of measurement) } & \multicolumn{2}{|c|}{ Zk } & \multicolumn{2}{|c|}{$\mathrm{Pb}$} & \multicolumn{2}{|c|}{ LG } \\
\hline & & LSM & SE & LSM & SE & LSM & SE \\
\hline \multirow[t]{4}{*}{ Open field test } & Probability of occurrence of defecation & $0.18^{B}$ & 0.04 & $0.08^{B}$ & 0.04 & $0.8^{\mathrm{A}}$ & 0.05 \\
\hline & Probability of occurrence of comfort behaviours & $0.22^{B}$ & 0.06 & $0.32^{B}$ & 0.06 & $0.62^{A}$ & 0.06 \\
\hline & Probability of occurrence of shake-off behaviour & $0.06^{B}$ & 0.04 & $0.18^{B}$ & 0.04 & $0.6^{\mathrm{A}}$ & 0.05 \\
\hline & Probability of occurrence of vocalisation & $0.08^{A}$ & 0.05 & $0.34^{B}$ & 0.05 & $0.36^{B}$ & 0.05 \\
\hline
\end{tabular}

Zk, Green-legged Partridge; Pb, Polbar; LG, Leghorn; LSM, lease squares means; SE, standard error.

${ }_{A, B}$ Means marked with different letters differ significantly between the breeds at $p \leq 0.001$.

to birds' behavioural needs [11]. The natural behaviours of domestic fowl, e.g. free movement, pecking, scratching the ground, wing flapping, self-grooming, or quiet rest and sleep, may be limited by the impossibility to express them. The tests carried out in this study were aimed at verification whether the many- generation hen selection, which indirectly determines birds' behaviour [12], exerted an effect on the behavioural variability and whether birds from different breeds characterised by dissimilar performance value exhibited different behaviours.

The results demonstrated such differences between the analysed breeds. Noteworthy is the relatively long time devoted by the Zk birds to explore the enriched feed; this parameter had several-fold higher values than in the case of the $\mathrm{Pb}$ and LG breeds. The birds did not only ingest the feed but also expressed the need for scratching and searching, thus satisfying one of the basic needs, i.e. curiosity [13]. However, it seems that the scratching and searching need is strongly developed mainly in the Zk birds, which represent primitive breeds that have not been selected towards high performance value. This element of the environment was not preferred by the hens from the other breeds. The LG hens, which are mainly reared as high-yield layers in intensive production, exhibited considerably greater interest in the commercial feed, which has a form of a homogeneous granulate and ingestion thereof does not require searching for edible parts. These results indicate that selection can significantly change hens' behaviour and food preferences. High-yield breeds are targeted at feed intake that will fulfil their physiological rather than behavioural needs. For economic reasons, bird breeds with the highest feed conversion rates and absence of the scratching and searching behaviour are preferred in breeding.

Another enriching element, i.e. the mirror, showed considerable differences in curiosity among the breeds. The longest

Table 6. Estimators of differences in traits defining birds' interest in the object depending on the breed, their significance and confidence interval

\begin{tabular}{|c|c|c|c|c|c|c|}
\hline Object & Breed & Breed & Estimate & Probability & Adjust lower & Adjust upper \\
\hline \multirow[t]{3}{*}{ Water (W) } & LG & $\mathrm{Pb}$ & -38.16 & 0.373 & -196.12 & 119.80 \\
\hline & LG & Zk & 5.41 & 0.899 & -152.54 & 163.36 \\
\hline & $\mathrm{Pb}$ & Zk & 43.57 & 0.309 & -114.44 & 201.58 \\
\hline \multirow[t]{3}{*}{ Commercial feed (CF) } & LG & $\mathrm{Pb}$ & 221.31 & 0.000 & 63.35 & 379.27 \\
\hline & $L G$ & Zk & 171.29 & 0.000 & 13.34 & 329.24 \\
\hline & $\mathrm{Pb}$ & Zk & -50.02 & 0.243 & -208.03 & 107.99 \\
\hline \multirow[t]{3}{*}{ Enriched feed (EF) } & LG & $\mathrm{Pb}$ & 21.17 & 0.621 & -136.79 & 179.13 \\
\hline & LG & Zk & -97.85 & 0.023 & -255.80 & 60.10 \\
\hline & $\mathrm{Pb}$ & Zk & -119.02 & 0.006 & -277.03 & 38.99 \\
\hline \multirow[t]{3}{*}{ Shelter (S) } & LG & $\mathrm{Pb}$ & 126.55 & 0.003 & -31.41 & 284.51 \\
\hline & LG & Zk & 43.22 & 0.313 & -114.73 & 201.17 \\
\hline & $\mathrm{Pb}$ & Zk & -83.33 & 0.050 & -241.34 & 74.68 \\
\hline \multirow[t]{3}{*}{ Mirror (M) } & LG & $\mathrm{Pb}$ & 163.93 & 0.000 & 5.97 & 321.89 \\
\hline & LG & Zk & 198.47 & 0.000 & 40.52 & 356.42 \\
\hline & $\mathrm{Pb}$ & Zk & 34.54 & 0.420 & -123.47 & 192.55 \\
\hline \multirow[t]{3}{*}{ Sandpit (SA) } & LG & $\mathrm{Pb}$ & -68.27 & 0.111 & -226.23 & 89.69 \\
\hline & LG & Zk & -1.76 & 0.967 & -159.71 & 156.19 \\
\hline & $\mathrm{Pb}$ & Zk & 66.51 & 0.121 & -91.50 & 224.52 \\
\hline \multirow[t]{3}{*}{ Floor (F) } & LG & $\mathrm{Pb}$ & -13.58 & 0.751 & -171.54 & 144.38 \\
\hline & LG & Zk & 104.49 & 1.000 & -53.46 & 262.44 \\
\hline & $\mathrm{Pb}$ & Zk & 118.07 & 0.769 & -39.94 & 276.08 \\
\hline
\end{tabular}

LG, Leghorn; Pb, Polbar; Zk, Green-legged Partridge. 
time for examination of the object was devoted by the LG hens. The birds pecked at their reflection in the mirror. The interest in the mirror may indicate great curiosity in this breed. It should be noted that this temperament feature cannot be fully satisfied in the farm rearing conditions. A stimulus-poor and monotonous environment does not offer opportunities to satisfy curiosity. Hence, it is possible that boredom and an attempt to satisfy curiosity is one of the causes of feather plucking, which is quite a common phenomenon in the LG breed (own unpublished observations).

There were also differences in the birds' interest in the shelter. This object was clearly avoided by the Pb breed. Simultaneously, none of the enriching elements was found to define the preferences of this breed, as in the case of Zk and LG. This may be a result of the origin and the components of this breed, i.e. heavy meat breeds and the primitive $\mathrm{Zk}$ breed. Hence, the behaviour of these hens is characterised by elements differing them from light layer breeds such as the LG and from the Zk breed. It can therefore be concluded that hybrids of layer breeds reared in a farm breeding system will differ in their preferences and behaviour from parent breeds. This is an important finding, as it indicates that the assessment of chicken behaviour cannot be limited to testing pure-bred birds.

There were differences in the behaviour of the analysed hen breeds in terms of emotional reactivity. Animals respond adequately to the degree of emotional arousal, which is strongly associated with the breed in addition to individual traits. The indicators of the emotional status comprise the locomotion speed, vocalisation, defecation, and self-grooming. The present investigations demonstrated differences in the level of these indicators depending on the breed. Within a similar locomotion time (no statistical differences), the LG hens covered a several-fold higher number of squares on the floor, which evidenced a fast locomotion rate. Additionally, there was a significantly greater probability of defecation in this breed. These indicators might suggest increased anxiety and higher fearfulness in LGs [14]; however, another group of behaviours associated with comfort activities (e.g. cleaning feathers, flapping wings, ruffling feather, scratching the body) was noted in this breed more often than in the others (Table 5). As reported by Zimmerman et al [14] increased locomotion is correlated with anxiety about an upcoming aversive event, whereas anticipation of a positive event is associated with comfort behaviours (e.g. cleaning feathers, flapping wings, ruffling feather, scratching the body). It should be underlined that application of a standard open-field test for assessment of fearfulness $[15,16]$ without modifications consisting in enrichment of the environment with additional elements would suggest a high level of fear in the LG hens. In this case, however, the presence of the interest in the elements of the environment with the absence of significant differences between the investigated breeds should be emphasised. Simultaneously, if the animal shows interest in objects, increased locomotion should not be regarded as expression of stress, as a stressed and terrified animal does not explore the environment. The relationships between the emotional reaction to the environment and the decision to avoid or approach the environment are key elements of animal welfare [17]. The behaviour of the LG hens indicates that the breed is characterised by very strong emotional arousal and high reactivity, but not necessarily fearfulness. The present results agree with investigations demonstrating that birds with white plumage exhibit higher emotional reactivity than birds with coloured feathers [18-20]. There were no differences in the level of reactivity between the coloured $\mathrm{Zk}$ and $\mathrm{Pb}$ breeds. This result should not be surprising, given the origin of the $\mathrm{Pb}$ breed. Importantly, only the LG breed has been intensively selected for many generations towards higher laying performance and it is currently characterised by very high laying rates. The $\mathrm{Zk}$ and $\mathrm{Pb}$ hens belong to conservative herds and constitute a genetic reserve; therefore, no selection targeted at birds' performance traits is being carried out. In conclusion, the higher reactivity of LG hens may also be a result of indirect selection and a behavioural response to the high physiological requirements of the organism.

The present investigations also included a tonic immobility test, whose results can be an indicator of the level of fearfulness, as suggested by various researchers $[8,21]$. Tonic immobility is an unconditioned reaction that can be easily induced manually and is reflected by bird's immobility and lower reactivity to external stimulation [9]. It is believed that the latency of the first head movement and the TI duration are positively correlated with fearfulness [22]. In the present study, the shortest duration of immobility was recorded in the $\mathrm{Pb}$ breed; however, the results of the open-field test do not show lower fearfulness in this breed in comparison to the others. There were no differences between the breeds in the time required by the birds to start exploration of the environment or in the locomotion time, which can undoubtedly be indicators of the level of fear [10]. TI is thought to be a behaviour protecting birds from predators $[23,24]$. Given this theory, it can be assumed that primitive behaviours related to species survival can be eliminated by selection. The LGs needed twice as long latency of tonic immobility, which did not influence the duration of immobility. Possibly, selection eliminates the physiological reaction of immobility but not its duration. The duration of immobility was the longest in the primitive Zk breed; yet, statistically significant differences were noted exclusively between $\mathrm{Zk}$ and $\mathrm{Pb}$, and not between the specialised LG breed. As indicated by the other research results, tonic immobility is difficult to achieve by excitable birds with increased emotional reactivity. Thus, the latency of immobility may be a specific marker of emotional excitability of an individual. 


\section{CONCLUSION}

To sum the study results, it can be concluded that the birds from the different breeds differ not only in the degree of excitation and emotional reactivity but, importantly, also in their preferences for environment-enriching elements. Ensuring well-being should therefore consider environmental modifications that will facilitate expression of characteristic and essential elements of the breed behaviour. The greatest emotional reactivity was detected in the LG breed, which may be a result of selection targeted at increasing chicken productivity [20]. The differences in the behaviour of birds from the analysed breeds indicate that laying hens cannot be regarded as one group of animals with the same environmental requirements. Therefore, establishment of welfare requirements should be based on verification of elements that are indeed essential for a particular genetic line to prevent disturbance in the homeostasis of the organism and to limit the occurrence of behavioural abnormalities.

\section{CONFLICT OF INTEREST}

We certify that there is no conflict of interest with any financial organization regarding the material discussed in the manuscript.

\section{REFERENCES}

1. Lay Jr DC, Fulton RM, Hester PY, et al. Hen welfare in different housing systems. Poult Sci 2011;90:278-94.

2. Janczak AM, Torjesen P, Palme R, Bakken M. Effects of stress in hens on the behaviour of their offspring. Appl Anim Behav Sci 2007;107:66-77.

3. Lindqvist $C$, Janczak AM, Natt D, et al. Transmission of stressinduced learning impairment and associated brain gene expression from parents to offspring in chickens. PLoS ONE 2007;2: 364.

4. Śmiechowska M, Podgórniak P. Study and assessment of selected quality parameters of organic hen eggs available on the tri-city market. J Agric Eng Res 2013;58:186-9.

5. Program for the protection of genetic resources of laying hens population. Annex No. 1 to the Order No. 16/07 from 1.06.2007.

6. Anang A, Mielenz N, Schüler L. Genetic and phenotypic parameters for monthly egg production in White Leghorn hens. J Anim Breed Genet 2000;117:407-15.

7. Rodenburg TB, Buitenhuis AJ, Ask B, et al. Heritability of feather pecking and open-field response of laying hens at two different ages. Poult Sci 2003;82:861-7.

8. Jones RB. The tonic immobility reaction of the domestic fowl: a review. Worlds Poult Sci J 1986;42:82-96.

9. Doğan Narinç D, Aygün A. A non parametric data transformation technique for quantitative genetic analyses: The rank transformation. AIP Conference Proceedings 2017;1833:020060.

10. Forkman B, Boissy A, Meunier-Salaün MC, Canali E, Jones RB. A critical review of fear tests used on cattle, pigs, sheep, poultry and horses. Physiol Behav 2007;92:340-74.

11. Bergmann S, Schwarzer A, Wilutzky K, et al. Behavior as welfare indicator for the rearing of broilers in an enriched husbandry environment-A field study. J Vet Behav 2017;19:90-101.

12. Rozempolska-Rucinska I, Kibala L, Prochniak T, Zieba G, Lukaszewicz M. Genetics of the novel object test outcome in laying hens. Appl Anim Behav Sci 2017;193:73-6.

13.Panksepp J. Affective consciousness: core emotional feelings in animals and humans. Conscious Cogn 2005;14:30-80.

14.Zimmerman PH, Buijs SAF, Bolhuis JE, Keeling LJ. Behaviour of domestic fowl in anticipation of positive and negative stimuli. Anim Behav 2011;81:569-77.

15. Jones RB, Waddington D. Modification of fear in domestic chicks, Gallus gallus domesticus, via regular handling and early environmental enrichment. Anim Behav 1992;43:1021-33.

16.de Haas EN, Kops MS, Bolhuis JE, Groothuis TG, Ellen ED, Rodenburg TB. The relation between fearfulness in young and stress-response in adult laying hens, on individual and group level. Physiol Behav 2012;107:433-9.

17. Barnard C. Ethical regulation and animal science: why animal behaviour is special. Anim Behav 2007;74:5-13.

18. Fraisse F, Cockrem JF. Corticosterone and fear behaviour in white and brown caged laying hens. Br Poult Sci 2006;47:1109.

19. Uitdehaag KA, Rodenburg TB, Van Reenen CG, et al. Effects of genetic origin and social environment on behavioral response to manual restraint and monoamine functioning in laying hens. Poult Sci 2011;90:1629-36.

20.de Haas EN, Kemp B, Bolhuis JE, Groothuis T, Rodenburg TB. Fear, stress, and feather pecking in commercial white and brown laying hen parent-stock flocks and their relationships with production parameters. Poult Sci 2013;92:2259-69.

21. Hocking PM, Channing CE, Waddington D, Jones RB. Agerelated changes in fear, sociality and pecking behaviours in two strains of laying hen. Br Poult Sci 2001;42:414-23.

22. Cockrem JF. Stress, corticosterone responses and avian personalities. J Ornithol 2007;148:169-78.

23. Gallup GC. Tonic immobility: the role of fear and predation. Psychol Rec 1977;27(Suppl 1):41-61.

24. Odén K. Fear and aggression in large flocks of laying hens effects of sex composition [doctoral thesis]. Skara, Sweden: Swedish University of Agricultural Sciences; 2003. 